\title{
Enhanced quantum efficiency of Ge solar cells by a two-dimensional photonic crystal nanostructured surface
}

\author{
I. Prieto, ${ }^{1}$ B. Galiana,${ }^{2}$ P. A. Postigo, ${ }^{1, a)}$ C. Algora,${ }^{2}$ L. J. Martínez, ${ }^{1}$ and I. Rey-Stolle ${ }^{2}$ \\ ${ }^{1}$ Instituto de Microelectrónica de Madrid, Centro Nacional de Microelectrónica, \\ Consejo Superior de Investigaciones Científicas, Isaac Newton 8, PTM Tres Cantos, 28760 Madrid, Spain \\ ${ }^{2}$ Instituto de Energía Solar-Universidad Politécnica de Madrid, E.T.S.I. Telecomunicación, \\ Avda. Complutense s/n, 28040 Madrid, Spain
}

(Received 2 July 2008; accepted 20 April 2009; published online 11 May 2009)

\begin{abstract}
A single-junction germanium solar cell with a photonic crystal nanostructured surface has been developed. The solar cell comprises a Ge $p$ - $n$ junction and an InGaP window layer. The InGaP window layer has been nanopatterned with an extended photonic crystal structure consisting on a triangular lattice of holes with submicronic sizes. Enhancements of the external quantum efficiency of $22 \%$ for a wide range of wavelengths and up to a $46 \%$ for specific wavelengths have been measured, which implies an increase in photocurrent between $11 \%$ and $22 \%$. A clear correlation with the area of photonic crystal patterned has been observed. (C) 2009 American Institute of Physics. [DOI: 10.1063/1.3133348]
\end{abstract}

During the last years, much attention has been paid to photonic crystals (PCs) ${ }^{1,2}$ for different applications. PCs and photonic bandgap materials have been also proposed and used for solar cells. ${ }^{3-6}$ Moreover, there has been an important amount of work on light extraction out of light-emitting diode devices by 2 D PCs. ${ }^{7-9}$ In a similar way, transmission of the incident light inside a device can be enhanced by a 2D-PC layer and there are a number of theoretical ${ }^{10-12}$ and experimental $^{13,14}$ works related to this. Nevertheless, little work has been done in the actual manufacture and characterization of a complete solar cell with a 2D PC on its front surface, conceived as a periodic distribution of the dielectric constant in the plane (the surface of the solar cell) and involving subwavelength motifs. In this case, the PC effect is slightly different from that in slabs or suspended membranes ${ }^{15}$ and lacks of the confinement in the vertical direction provided by total internal reflection. Despite the partial vertical confinement, all the above mentioned reasons can justify to try to use a PC front surface with subwavelength motifs. Consequently, the aim of this work is to prove the validity of a 2D PC as an additional strategy to improve solar cells performance. With this aim, we have manufactured a single $p$ - $n$ junction solar cell based on Ge with a PC nanopatterned InGaP window layer. In order to quantify the improvement introduced by the PC, the external quantum efficiency (EQE) and the $I-V$ curves under illumination of the solar cells manufactured in the same batch with and without the PC have been measured. We have found an increase in $\mathrm{EQE}$ around $22 \%$ in average for the solar cells with the PC structure, which in terms of photocurrent implies an increase between $16 \%$ and $25 \%$ for direct terrestrial spectrum (AM1.5D).

A layout of the device is shown in Fig. 1. The solar cell is composed by a single $p$ - $n$ junction formed by the diffusion of phosphorus to the $p$-type Ge substrate during the metalorganic vapor phase epitaxy growth of a GaInP window layer. Ge wafers, $150 \mu \mathrm{m}$ thick, (100) $6^{\circ}$ off toward (111) have been used as substrates. The thickness of the InGaP

${ }^{a)}$ Electronic mail: aitor@imm.cnm.csic.es. window layer is $1000 \mathrm{~nm}$ and it is $n$-type doped with $\mathrm{Si}$ with a concentration $N_{D}=1 \times 10^{18} \mathrm{~cm}^{-3}$. A GaAs cap layer has been grown to ease the front Ohmic contact. The back contact is carried out with Au. Front Ohmic contacts by deposition of the AuGe-Ni-Au system outside of a circular pattern made by photolithographic techniques were performed. No antireflection coating has been deposited. The whole diameter of the uncovered front surface ranges from 1.4 to 1.6 $\mathrm{mm}$ on different devices with zero shadowing factor (no fingers have been defined). The major advantage of a PC window layer is, in principle, to introduce diffraction, where the photon momentum (k) can be scattered away from the specular direction with $\left(k_{\|}=k_{i \|}+\mathbf{G}\right)$, where $\mathbf{G}$ is a reciprocal lattice vector and $\mathbf{k}_{i}$ is the incident wave vector. The dielectric contrast of the air to $\operatorname{InGaP}(\sim 3)$ is large enough for sufficient diffraction of light by the PC. Maximum diffraction is expected when the Fourier components of the dielectric function of the 2D PC is a maximum. Moreover, it has been shown ${ }^{16}$ that discrete spots of diffraction pattern in a weakly modulated PC, including those of conventional diffraction gratings, become continuous when the dielectric modulation becomes finite. Furthermore, in a finitemodulated PC, the diffraction can take place even in the

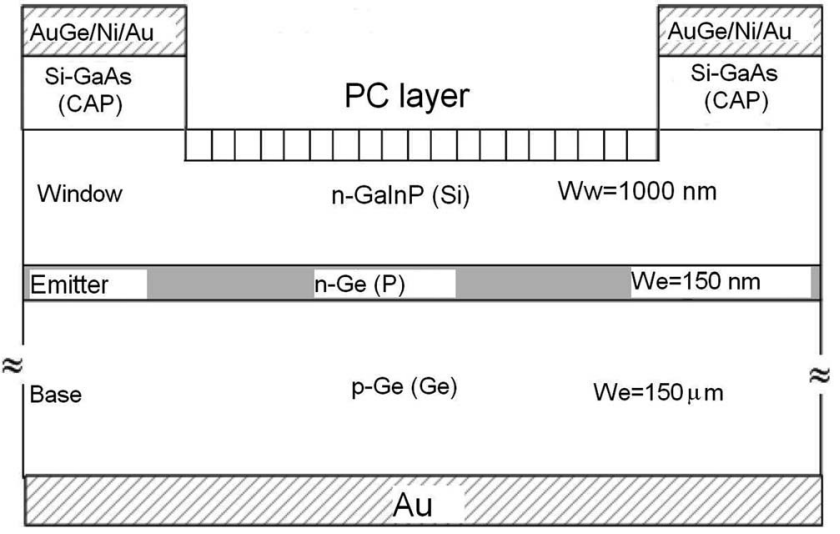

FIG. 1. Layout of the cross section of the solar cell patterned with the PC layer formed by a triangular lattice of holes with radius $r=200 \mathrm{~nm}$, lattice parameter $a=600 \mathrm{~nm}$, and depth $d=300 \mathrm{~nm}$. 
region prohibited by Bragg's law producing available states for the incident light, which are evanescent in the case of a diffraction grating.

Using a triangular lattice of holes of radius $r$ and lattice parameter $a$, a value of $r / a \sim 0.3$ maximizes the strength of the first Fourier component $(2 \pi / a) .{ }^{11}$ We take this value to fabricate a subwavelength periodic pattern (a PC in the horizontal plane) and to measure its actual performance. Nevertheless this design may need of careful optimization for future devices. The depth of the holes for the top transmission PC grating $(d)$ needs to be further optimized but a good starting value is for $d / a$ around 0.5 . Since this value depends on the wavelength, we have chosen an optimized thickness in average for the spectral region near the band edge (800$1600 \mathrm{~nm}$ ). This is because the main applications of Ge solar cells are as forming part of $\mathrm{GaInP} / \mathrm{GaInAs} / \mathrm{Ge}$ triple-junction cells and for thermophotovoltaic applications. In both cases, the best performance of the Ge cell is pretended in the infrared. As a rule of thumb the lattice spacing should be near the wavelength of diffracted light inside the InGaP. However values of $a$ can still offer improvement and may be more convenient for fabrication. For diffraction to the PC, it is necessary for $a$ to be larger than the wavelength of light in the absorber layer $(a>\lambda / n)$ where $n$ is the index of refraction. We have used an averaged $n=3.3$ for the $\mathrm{InGaP}$ in the spectral region considered $(600-1800 \mathrm{~nm})$. Since the maximum is around $1850 \mathrm{~nm}$, the above condition is well satisfied for $a=600 \mathrm{~nm}$. It is necessary to have several diffraction resonances within the wavelength window below the band edge, where the absorption length of photons is longer than the absorber layer thickness. Under all of these assumptions, the optimized solar cell with the PC can reduce considerably its reflection than without the PC. ${ }^{11,12}$

We have manufactured the PC nanopattern on the front exposed surface of the device. For this purpose we have employed electron-beam lithography over an extended area of a square about $1 \times 1 \mathrm{~mm}^{2}$. The PC pattern is made on a poly-methyl-methacrylate (PMMA) layer deposited by spincoating. The PC pattern is made by circular holes of radius $r=200 \mathrm{~nm}$ and a separation (lattice parameter) of $a$ $=600 \mathrm{~nm}$. The holes are distributed following a triangularsymmetry lattice and inside squares of $200 \times 200 \mu \mathrm{m}^{2}$ separated $20 \mu \mathrm{m}$ each. This is done to eliminate any photonic induced effect by misalignment during stitching of the writing fields. Also, the PC pattern is placed well inside the circular exposed surface, avoiding border effects or possible damage to the electrical contacts. In this way around a $60 \%$ of the total surface of the solar cell is patterned. After developing of the PMMA resist, the pattern is transferred to the InGaP layer by Ar-based reactive ion beam etching. The deep of the etched holes is $200 \mathrm{~nm}$. Finally, the remains of the PMMA layer are eliminated by oxygen-plasma etching. Figure 2 shows a microscope picture of the device with an inset showing a scanning electron microscope (SEM) picture of the PC nanopatterned structure. Solar cells with and without the PC pattern are fabricated together in order to facilitate experimental comparison. Nonhomogeneities due to change in material or processing technology are inexistent on these structures.

$I-V$ curves under illumination and EQE measurements have been carried out in a monochromator-based system with a light-beam chopper. The primary light source is a $1000 \mathrm{~W}$

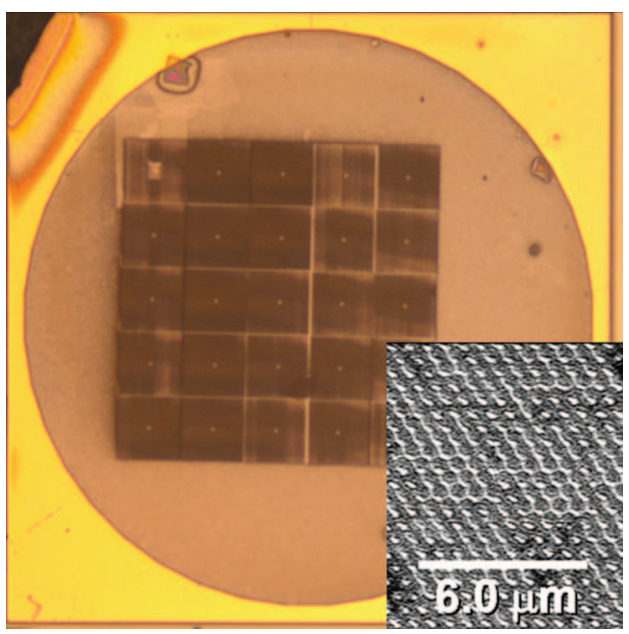

FIG. 2. (Color) Microscope picture of the solar cell device with the area patterned with a PC $\left(1 \mathrm{~mm}^{2}\right)$. Inset shows a SEM picture of the PC nanopatterned structure.

Xe lamp. The system has a monitor cell to assess the realtime fluctuations of the lamp. Both the monitor cell and the test cell are connected to two independent lock-in amplifiers, which permits the simultaneous measurement of both the monitor cell and the reference/test cell. This setup allows the minimization of the measurement errors resulting from lamp fluctuations, very significant in certain spectral ranges. The chuck for the test cell is temperature controlled by a Peltier element. The spectral range goes from 300 to $2000 \mathrm{~nm}$ but the relevant range for the cell is between 600 and $1800 \mathrm{~nm}$. In order to calibrate the system status at the beginning of every measurements session, a GaSb solar cell is used as reference. The spot light impinging the cells was in all cases of $1.2 \mathrm{~mm}$ of diameter.

Figure 3 shows the $I-V$ curves under illumination of two solar cells, one with PC pattern and one without it, otherwise identical. Despite the intrinsic performance of the used solar cells as not optimum (filling factors around 52\%) due to their experimental nature (these cells were obtained with heteroepitaxial techniques), there is an improvement of the efficiency of about $11 \%$ for the cell with PC pattern. In this prototype, only a $40 \%$ of the surface was patterned with the PC. By linear extrapolation, an enhancement of 25\%-26\%

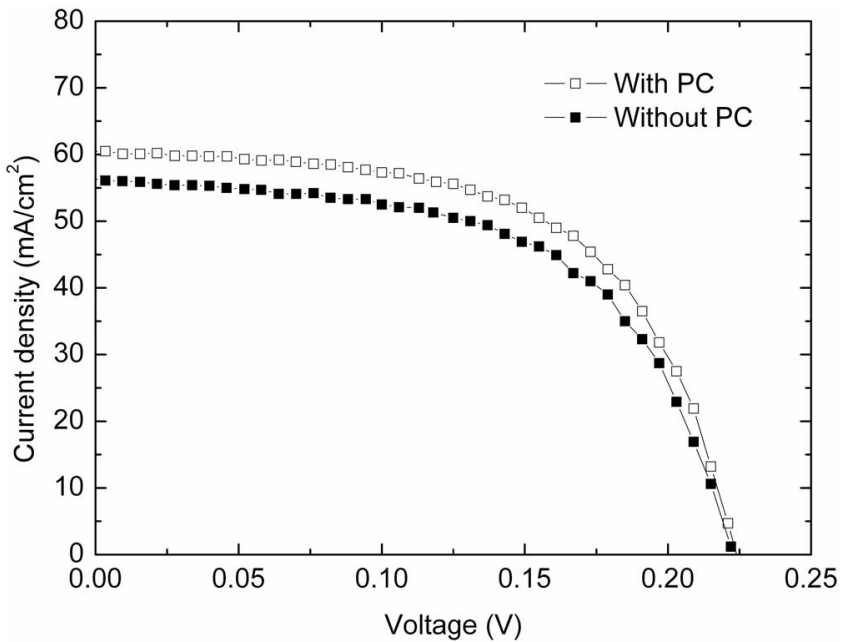

FIG. 3. $I-V$ curves under illumination for PC patterned and nonpatterned solar cells. 


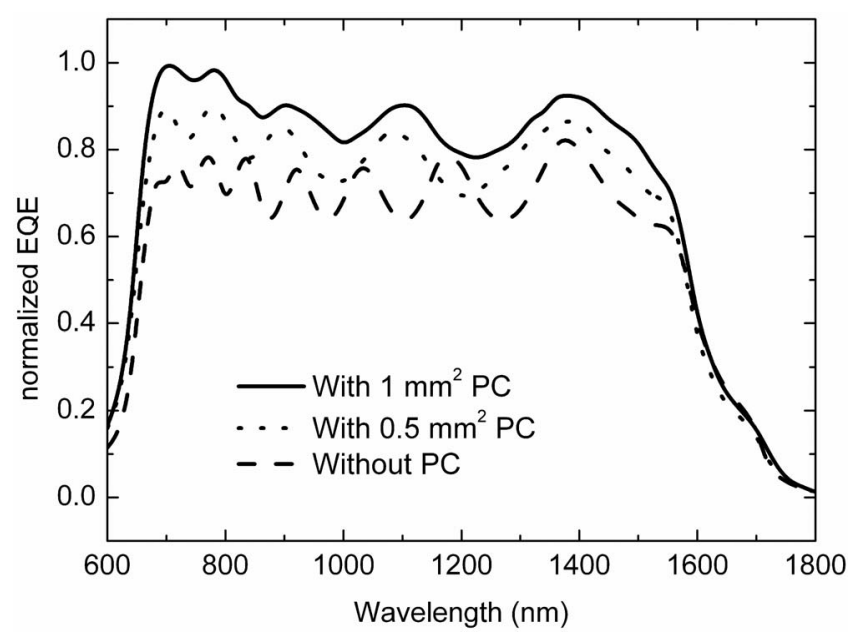

FIG. 4. Relative EQE for two PC-patterned solar cells with two different areas patterned, $1 \mathrm{~mm}^{2}$ (open triangles) and $0.5 \mathrm{~mm}^{2}$ (gray circles) and for a nonpatterned solar cell of the same size (black curve).

can be obtained for a solar cell with the entire surface patterned by a PC. The measured increment in the photocurrent is $8 \%$, which gives an enhancement in the photocurrent of $22 \%$ for a fully covered surface. The open-circuit voltage $\left(V_{\mathrm{OC}}\right)$ was not degraded. Also, the dark $I-V$ curves of both devices, with and without PC were observed to be exactly the same, which implied that none degradation has been introduced to the device due to the PC pattern fabrication.

Figure 4 shows the relative EQE for two PC-patterned solar cells with two different areas patterned, $1 \mathrm{~mm}^{2}$ and $0.5 \mathrm{~mm}^{2}$. The experimental EQE data have been normalized to the highest EQE value. Figure 3 shows also the EQE for a nonpatterned solar cell of the same size. The EQE in both the PC-patterned solar cells are always higher than in the nonpatterned cell. For the cell with PC-pattern covering $1 \mathrm{~mm}^{2}$ the enhancement of current using the spectrum AM1.5D is $25 \%$, whereas for the cell with half of the area patterned the increase is $16 \%$. Figure 5 shows the reflectivity measured in the solar cell with the $1 \mathrm{~mm}^{2}$ patterned PC and in the bare solar cell. The size of the spot is around $250 \mu \mathrm{m}$ in diam-

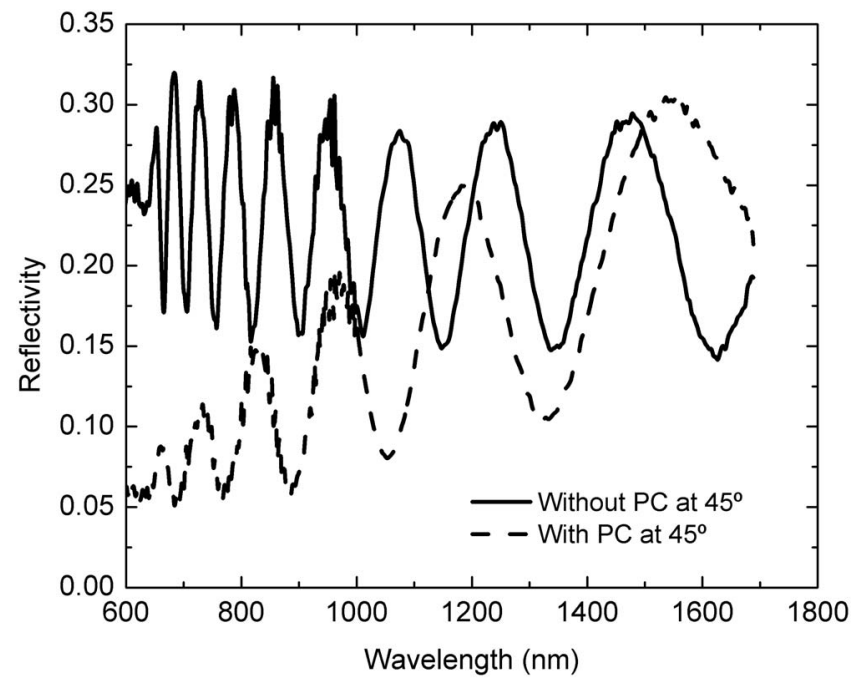

FIG. 5. Reflectivity of the PC-patterned solar cell and the nonpatterned reference cell. eter. The reflectivity measured at $45^{\circ}$ incidence is smaller for the PC solar cell for most of the wavelengths. Measurements performed at normal incidence give very similar values and are not included.

All of this shows the validity of our approach to enhance the performance of solar cells, which could be also applied to other photon-sensing devices such as photodetectors and thermophotovoltaic devices. Further work is needed to understand in deep all the possible photonic effects involved, such as subwavelength diffraction, variation of the index of refraction due to the photonic bands, change of the lifetime of the photons by the PC layer, or role of the submicronic roughness produced during the etching. Moreover, especial efforts should be made in order to find optimized PC patterns for the above mentioned devices.

In summary, we have manufactured a single-junction $\mathrm{Ge} / \mathrm{InGaP}$ solar cell with a PC nanostructured surface. An increase in the photocurrent up to $8 \%$ has been achieved on a solar cell with $40 \%$ of its surface covered with a PC pattern. Enhancements of the EQE of $22 \%$ for a wide range of wavelengths and up to $46 \%$ for specific wavelengths have been measured. A correlation with the area of PC patterned has been observed. Further work is needed for the optimization of the PC pattern and to asses the advantages of using a PC-pattern versus antireflection coatings, which can be compatible with the PC structure, and to establish its performance with the angle of incidence.

The authors would like to acknowledge support from projects NAN2004-08843-C05-04, NAN2004-09109-C0401, TEC-2005-05781-C03-01, CONSOLIDER-Ingenio 2010 "Genesis-FV" project (Project No. CSD2006004), TEC200502745. TEC200422300E, NUMANCIA (Grant No. S05050/ENE/0310).

${ }^{1}$ E. Yablonovitch, Phys. Rev. Lett. 58, 2059 (1987); S. John, ibid. 58, 2486 (1987).

${ }^{2}$ K. Ohtaka, Phys. Rev. B 19, 5057 (1979).

${ }^{3}$ A. Mihi, M. E. Calvo, J. A. Anta, and H. Miguez, J. Phys. Chem. C 112, 13 (2008).

${ }^{4}$ P. Bermel, C. Luo, L. Zeng, L. C. Kimerling, and J. D. Joannopoulos, Opt. Express 15, 16986 (2007).

${ }^{5}$ L. Zeng, Y. Yi, C. Hong, J. Liu, N. Feng, X. Duan, L. C. Kimmerling, and B. A. Alamariu, Appl. Phys. Lett. 89, 111111 (2006).

${ }^{6}$ C. Seassal, Y. Park, A. Fave, E. Drouard, E. Fourmond, A. Kaminski, M. Lemiti, X. Letartre, and P. Viktorovitch, Proc. SPIE 7002, 700207 (2008). ${ }^{7}$ B. S. Cheng, C. H. Chiu, K. J. Huang, C. F. Lai, H. C. Kuo, C. H. Lin, T. C. Lu, S. C. Wang, and C. C. Yu, Semicond. Sci. Technol. 23, 055002 (2008).

${ }^{8}$ J. Shakya, K. H. Kim, J. Y. Lin, and H. X. Jiang, Appl. Phys. Lett. 85, 142 (2004).

${ }^{9}$ K.-H. Kim, J.-H. Shin, N.-M. Park, C. Huh, T.-Y. Kim, K.-S. Cho, J. C Hong, and G. Y. Sung, Appl. Phys. Lett. 89, 191120 (2006).

${ }^{10}$ P. S. Dutta, Proceedings of the 2007 International Workshop on Physics of Semiconductor Devices (IWPSD '07) (2007), p. 828.

${ }^{11}$ R. Biswas and D. Zhou, Mater. Res. Soc. Symp. Proc. 989, A3.2 (2007); Proceedings of the Amorphous and Polycrystalline Thin-Film Silicon Science and Technology (2007), pp. 35-40.

${ }^{12}$ K. R. Catchpole and M. A. Green, J. Appl. Phys. 101, 063105 (2007).

${ }^{13}$ Y. Kanamori, M. Sasaki, and K. Hane, Opt. Lett. 24, 1422 (1999).

${ }^{14}$ Y. Kanamori, E. Roy, and Y. Chen, Microelectron. Eng. 78, 287 (2005).

${ }^{15}$ S. G. Johnson, Phys. Rev. B 60, 5751 (1999).

${ }^{16}$ G. Alagappan, X. W. Sun, and M. B. Yu, J. Opt. Soc. Am. A Opt. Image Sci. Vis. 25, 1098 (2008) 